\title{
Classification Scheme Item Relationship
}

National Cancer Institute

\section{Source}

National Cancer Institute. Classification Scheme Item Relationship. NCI Thesaurus. Code C42632.

The affiliation between two occurrences of Classification Scheme Items. 